\title{
Long-Term Potentiation in the Hippocampus Using Depolarizing Current Pulses as the Conditioning Stimulus to Single Volley Synaptic Potentials
}

\author{
B. Gustafsson, H. Wigström, W. C. Abraham, ${ }^{a}$ and Y.-Y. Huang \\ Department of Physiology, University of Göteborg, S-400 33, Göteborg, Sweden
}

The conditions responsible for the associative properties of long-term potentiation (LTP) were examined in the CA1 region of the hippocampal slice preparation. Intracellularly recorded EPSPS resulting from single-volley stimulation at low frequency $(0.15-0.1 \mathrm{~Hz})$ in the stratum radiatum or oriens were paired with depolarizing current pulses (50-100 msec) injected through the recording microelectrode. It is shown that these EPSPs, when paired with pulses of sufficient magnitude, become potentiated. This potentiation generally reached a peak after 20-30 pairing events and could outlast the conditioning period by more than $1 \mathrm{hr}$. It was specific to the paired input, was blocked by 2-amino-5-phosphonovalerate (APV) and was largely blocked by prior homosynaptic tetanization (and vice versa). In experiments performed with picrotoxin (PTX) in the bath, EPSPs were potentiated using 2-4 nA current pulses, with somewhat higher values in normal solution. The effective current pulses, in both normal and PTX solution, produced a repetitive spike discharge of 7-11 spikes (per $100 \mathrm{msec}$ ), and within this range, higher frequencies were associated with larger potentiations. However, since similar degrees of EPSP potentiation were observed following blockade of spike activity by intracellular QX-314, spike activity was not the primary conditioning factor. For the potentiation to appear, the EPSP had to occur together with the current pulse or precede it by less than about 100 msec. No potentiation was observed when the EPSP immediately succeeded the pulse. The results suggest that the cooperativity aspect of LTP is related to a need for sufficient postsynaptic depolarization. They also support the proposal that LTP is induced as a consequence of opening synaptic $N$-methyl-D-aspartate (NMDA) receptor channels.

An important characteristic of hippocampal long-term potentiation (LTP) is that it exhibits cooperativity, i.e., the degree of LTP produced is related to the number of coactive afferents (McNaughton ct al., 1978; Levy and Steward, 1979; Lee, 1983).

\footnotetext{
Received May 12, 1986; revised Aug. 9, 1986; accepted Aug. 28, 1986.

This work was supported by the Swedish Medical Research Council (projects 05180 and 05954), the Swedish Natural Science Research Council (project 4018), Magnus Bergvalls Stiftelse, and Stiftelsen Lars Hiertas Minne. We thank Astra Läkemedel, Södertälje, for the generous gift of QX-314. W.C.A. was supported by a NATO postdoctoral fellowship, and Y.-Y.H. by the China Education Ministry.

Correspondence should be addressed to Dr. B. Gustafsson, Department of Physiology, P.O. Box 33031, S-400 33, Göteborg, Sweden.

a Present address: Department of Psychology, University of Otago, Box 56, Dunedin, New Zealand.

Copyright (C) 1987 Society for Neuroscience $0270-6474 / 87 / 030774-07 \$ 02.00 / 0$
}

This property gives an associative dimension to LTP and conforms with the suggestion that LTP is a possible neuronal substrate for learning and memory (e.g., Teyler and Discenna, 1984). While various suggestions have been forwarded to explain cooperativity (McNaughton et al., 1978; Eccles, 1983; Levy and Steward, 1983; Hopkins and Johnston, 1984), it remains undecided whether it is related to interactions occurring pre- or postsynaptically. One recently proposed explanation of LTP induction (Wigström and Gustafsson, 1985a) holds that LTP is produced as a consequence of an opening of voltage-sensitive synaptic channels located postsynaptically in the spine membrane and that cooperativity results from the need for sufficient depolarization at these sites. A key prediction in this proposal is that the generation of LTP does not require tetanic activation of a test input but will occur when single afferent volleys are present during periods of large postsynaptic depolarization produced in some other manner. An indication of such behavior was obtained by pairing single volleys with brief tetani to a separate input to produce LTP (Gustafsson and Wigström, 1986). A more definitive demonstration, however, would be to show that LTP is induced when single volleys are paired with postsynaptic depolarization generated by current injection from an intracellular micropipette, and the present study is concerned with this question. A preliminary note of the present findings has been published (Wigström et al., 1986).

\section{Materials and Methods}

Experiments were performed on guinea pig hippocampal slices (Skrede and Westgaard, 1971). The animals were anesthetized with ether and their brains removed. The hippocampus on one side was dissected out and cut into $400-\mu \mathrm{m}$-thick transverse slices. During the experiment, the slices were kept on a nylon net in a constant-flow incubation chamber at $30-32^{\circ} \mathrm{C}$, half-submerged by a solution containing the following (in $\mathrm{mM}$ ): $\mathrm{NaCl}, 124 ; \mathrm{KCl}, 4 ; \mathrm{CaCl}_{2}, 4 ; \mathrm{MgCl}_{2}, 4 ; \mathrm{NaHCO}_{3}, 26 ;$ glucose, 10. Concentrations of calcium and magnesium in the solution were somewhat higher than normal to partially counteract the excitability increase obtained in the experiments in which picrotoxin (PTX) was present in the bath.

CA1 pyramidal cells were synaptically activated by electrical stimulation of afferent fibers in stratum radiatum or oriens (Fig. 1B). Monopolar cathodal stimulation $(0.1 \mathrm{msec}, 5-30 \mu \mathrm{A})$ was delivered through electrolytically sharpened tungsten wires. Recordings were obtained extracellularly from the dendritic layers using glass micropipettes filled with $3 \mathrm{M} \mathrm{NaCl}(3-10 \mathrm{M} \Omega$ ). Intracellular recordings from pyramidal cells were made using micropipettes filled with either $2 \mathrm{~m}$ K-methylsulfate, $4 \mathrm{~m} \mathrm{~K}$-acetate, or, in experiments on PTX-treated slices, $3 \mathrm{M} \mathrm{KCl}$. In some experiments, the local anesthetic QX-314 (Astra) was added to the recording solution to block fast sodium-dependent action potentials (Connors and Prince, 1982). Cells accepted for analysis had resting membrane potentials greater than $-60 \mathrm{mV}$ and input resistances exceeding $20 \mathrm{M} \Omega$. After passing through a high-impedance preamplifier 

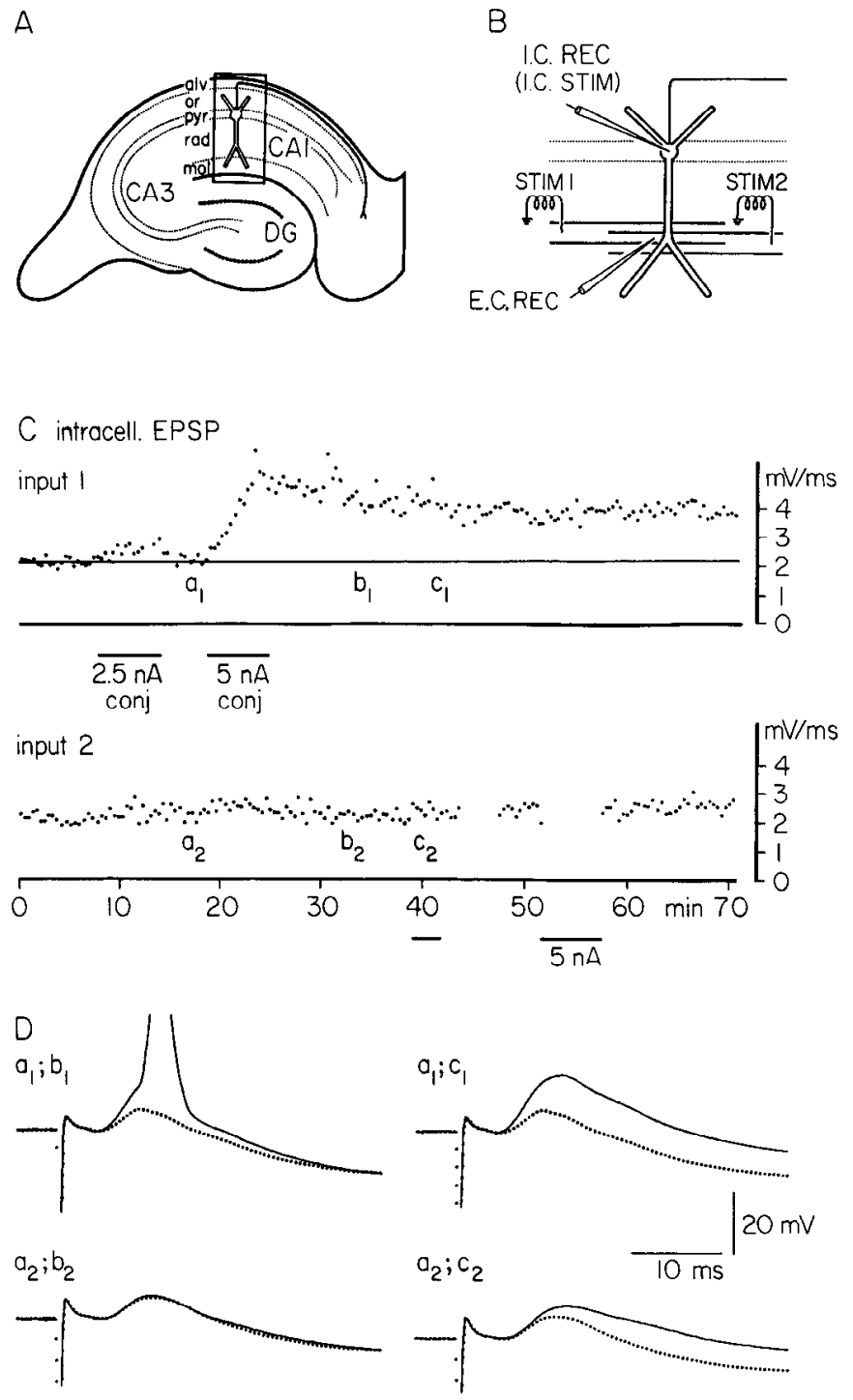

Figure 1. EPSP potentiation induced by combining single afferent volleys and depolarizing current pulses. $A$, Schematic drawing of the hippocampal slice showing the CA1 region studied (boxed area). Abbreviations: alv, alveus; or, stratum oriens; $p y r$, stratum pyramidale; rad, stratum radiatum; mol, stratum moleculare. $B$, Diagram showing the arrangement of stimulating and recording electrodes. $C$, Measurements of the initial slopes of the intracellular EPSPs resulting from activation of input 1 (STIM 1) and input 2 (STIM 2) are shown for a series of test responses. Each point in this and all following graphs represents the average of 3 consecutive measurements. Each input was stimulated at a frequency of $0.1 \mathrm{~Hz}$, and the stimuli to STIM 1 and STIM 2 were kept $5 \mathrm{sec}$ apart. During the time indicated by the horizontal bars, each EPSP evoked by STIM 1 was followed by a $100 \mathrm{msec}$ depolarizing current pulse of the indicated magnitude starting just after the EPSP peak (cf. Fig. $3 B$ ). The delay to STIM 2 was increased by $100 \mathrm{msec}$ on 2 occasions, which accounts for the 2 interruptions in the input 2 measurements. On the second occasion, each EPSP evoked by STIM 2 was preceded by a $100 \mathrm{msec}$ depolarizing current pulse of the indicated magnitude, which thus ended just before EPSP onset. $D$, Averaged records $(n=10)$ taken at indicated times. Recordings $c_{1}$ and $c_{2}$ were taken during injection of $0.15 \mathrm{nA} \mathrm{DC}$ hyperpolarizing current to avoid spike generation (period indicated by bar in $C$ ). Note that this hyperpolarization increased the peak voltage of the control input (input 2) but not its initial slope. The experiment was performed in normal solution with no PTX present.
(Gustafsson et al., 1982), the signals were connected to an analog-todigital converter and fed into a NORD-100 minicomputer. The latter was used for on-line signal averaging and for measurement of waveform parameters. Extra- and intracellular EPSPs were measured as the slope of the initial rising phase of the corresponding potential. The computed waveform parameters, as well as digital records of original single or averaged potentials, were stored in the mass memory of the computer for later off-line analysis. Hard copies of experimental data were generated by a pen plotter. The membrane potential of the impaled cells was monitored from a continuous DC recording on a Grass polygraph.

\section{Results}

Using extracellular recording, it was previously shown that LTP of a test input could be induced by several pairings of a single afferent volley with a brief tetanus to separate afferents projecting to the same neurons (Gustafsson and Wigström, 1986). This effect of the conditioning train was tentatively ascribed to the postsynaptic depolarization generated by it. Figure $1 C$ shows that, in an intracellular recording from a pyramidal neuron, a single-volley EPSP (input 1) increased in magnitude (initial slope) when paired (cf. Fig. $3 B$ ) at low frequency $(0.1 \mathrm{~Hz}$ ) with a 100 msec, $5 \mathrm{nA}$ depolarizing current pulse. This increased EPSP generally reached a maximum after 20-30 conjunction events. Following the conjunction, the EPSP increase in this cell first decayed for some minutes but thereafter attained a more stable value, lasting throughout the $45 \mathrm{~min}$ observation period. This change in EPSP was always restricted to the input that was paired with the pulse, no change being observed in the other input (see input 2 in Fig. 1C) that in each experiment was alternated with the conditioned one.

In slices kept in normal solution, 51 inputs from 33 cells (17 experiments) were tested in this manner, and EPSP increases were observed for 37 of these inputs. In Fig. $2 A$ (open circles) the relative EPSP increase reached during the conjunction procedure is plotted in relation to the magnitude of the $100 \mathrm{msec}$ current pulse for these positive cases. For most cells, only a single current value was used, usually $5 \mathrm{nA}$, and, as can be observed in the plot, this pulse was generally sufficient to produce a large EPSP increase, $76 \%$ on average. In the 2 negative cases, larger currents $(8 \mathrm{nA})$ did produce EPSP increases of 22 and $35 \%$, respectively. Furthermore, in 3 cells, a current increment from 5 to $7 \mathrm{nA}$ caused an additional EPSP increase, 25$50 \%$ above that obtained by $5 \mathrm{nA}$. The average value of $76 \%$ is thus likely an underestimation of the maximal pairing effect. When the pairing was first started with lower currents of 2-3 $\mathrm{nA}$, the EPSP increase was considerably smaller than with $5 \mathrm{nA}$ or was nonexistent. This can be seen for input 1 in Figure $1 C$, where a $2.5 \mathrm{nA}$ pulse gave only a small effect, while a $5 \mathrm{nA}$ pulse produced a considerable increase (see also Fig. $3 A$ ).

As indicated above, no conjunction effects were observed for 14 inputs even with 7-8 nA pulses. These inputs were subsequently homosynaptically tetanized, but essentially no LTP of the intracellular EPSPs was observed 5-10 min after tetanization, despite large increases in the field EPSPs. The lack of conjunction cffect may reflect the trauma of electrode penetration in these cells (cf. Wigström et al., 1982).

In 8 additional experiments, PTX $(0.1 \mathrm{~mm})$ was present in the bath, and the relation between the EPSP increase during conjunction and the current magnitude in these experiments is also plotted in Figure $2 A$ (closed circles). The effect of pairing was tested on 29 inputs from 16 cells, and EPSP increases were observed for 26 of these inputs, the negative cases, as above, showing essentially no LTP with homosynaptic tetanization. 
$\wedge$

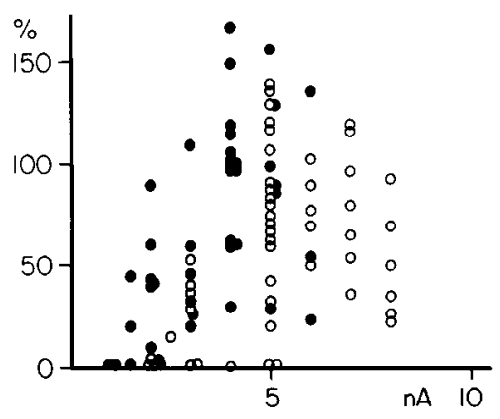

Compared to the results in normal solution, EPSP increases were now induced by somewhat lower currẹts, 2-3 nA pulses being generally effective, and $4 \mathrm{nA}$ giving an average $98 \%$ increase, i.e., a greater effect than $5 \mathrm{nA}$ in normal solution.

While pulses of about $100 \mathrm{msec}$ were used for the data described above, shorter current pulses $(30-50 \mathrm{msec}$, tested in PTX-treated slices) were also effective. For 7 inputs, such pulses (4-6 $\mathrm{nA}$ ) produced an average $82 \%$ EPSP increase during conjunction, i.e., an effect comparable to that given by the $100 \mathrm{msec}$ pulses. However, since in 3 of 4 inputs tested $100 \mathrm{msec}$ pulses had somewhat larger effects than the shorter ones, this longer duration was preferrcd as a standard. An increase of pulse duration from 100 to $200 \mathrm{msec}$ was also tested for one input, but no additional EPSP increase was produced.

\section{Relation between EPSP increase and spike activity}

The current pulses used for the pairing generally gave rise to a regular repetitive spike activity (Fig. $3 B$ ). The relation between the relative EPSP increase reached during the conjunction procedure and the number of spikes per $100 \mathrm{msec}$ is plotted in Figure $2 B$ for slices kept in normal solution (open circles) and in PTX-treated slices (closed circles). All tested inputs are not represented in this graph since it was not always possible to obtain the spike number value, mostly because of large changes in electrode resistance during the current injection. It is apparent from the graph that the values from normal and PTX-treated slices overlap considerably. Moreover, it appears that the current pulse must not only be strong enough to evoke spike activity, but the number of spikes must exceed 5-6/100 msec. The average effect increased from $25 \%$ for 7 spikes and $49 \%$ for 8 spikes to 64,82 , and $95 \%$ for 9,10 , and 11 spikes, respectively, when combining the values from the 2 different preparations.

To determine whether spike activity per se was important for the conjunction effect, experiments were performed with the local anesthetic QX-314 added to the solution in the recording micropipette. Depolarizing current pulses of $5 \mathrm{nA}$ now gave rise to membrane potential responses consisting of 1-2 prolonged spike responses; these were likely related to activation of voltage-dependent calcium channels. In conjunction tests performed for 19 inputs from 15 cells, EPSP increased in 13 . The relative increases during the conjunction procedure for these cases averaged $94 \%$, i.e., similar to that observed in cells having normal spike activity. In the negative cases, homosynaptic tetanization produced little or no LTP (see above).

\section{Duration of the pairing-induced EPSP increase}

After conjunction stimulation, the EPSP increase started to decay, and the value obtained $10 \mathrm{~min}$ later averaged $57 \%$ of the

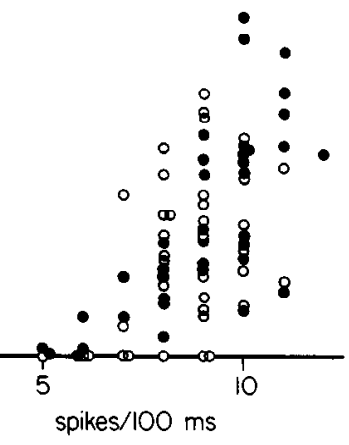

Figure 2. Relative amount of conjunction-induced potentiation versus current injected and spike frequency. $A$, Maximal increase in EPSP reached during the conjunction procedure plotted against the magnitude of the current pulse. Values obtained in normal solution are shown as open circles, those in the presence of PTX $(0.1 \mathrm{~mm})$ as filled circles. $B$, Same as in $A$, but the abscissa represents the number of spikes appearing per $100 \mathrm{msec}$ of current injection.

peak value. This value was obtained from 44 of the 76 tested inputs; the values from the remaining inputs are missing due to either the introduction of a homosynaptic tetanization during or soon after the conjunction (see below) or changes in the recording conditions. In the example illustrated in Figure 1, the EPSP increase remained fairly constant following this initial decay, and a similar result was observed in 12 of 23 inputs that were followed for $30 \mathrm{~min}$ or more after conjunction. For the remaining 11 inputs the EPSP continued to decay, essentially returning to baseline $15-30 \mathrm{~min}$ following conjunction. The initial decay for these 11 inputs was also more pronounced than

A

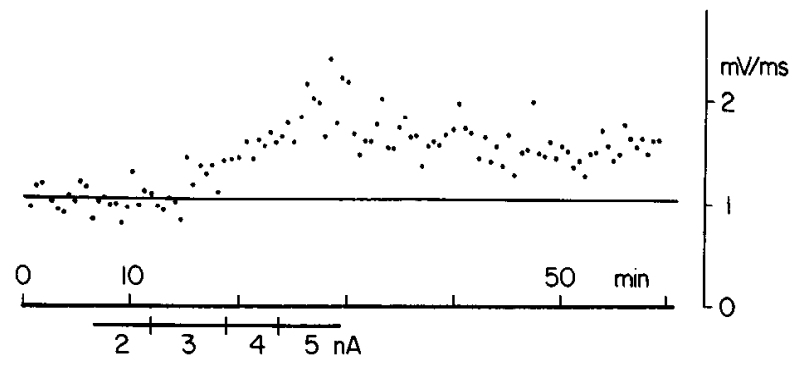

$\mathrm{B}$

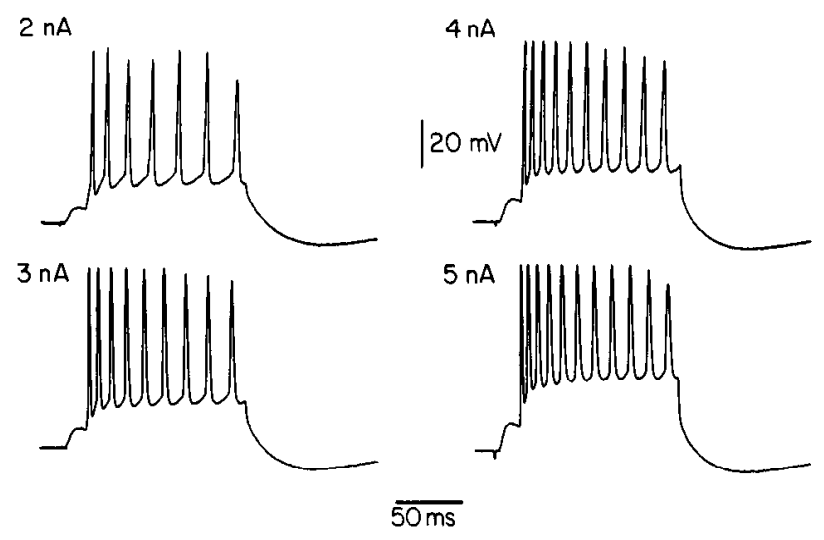

Figure 3. Relation between EPSP potentiation, current strength, and spike frequency for a single pyramidal neuron. $A$, Measurements of the initial slope of the intracellular EPSP are shown for a series of test responses. During the time indicated by bars, each EPSP was followed by a $100 \mathrm{msec}$ current pulse of the indicated magnitude. $B$, Averaged records $(n=10)$ taken at the indicated times. Note that since the records are averaged, spike height is also a function of the temporal dispersion of the spike potentials. Records were taken with the cathode follower in sample-and-hold mode $(3 \mathrm{kHz})$. The experiment was performed with 0.1 mM PTX in the bath. 


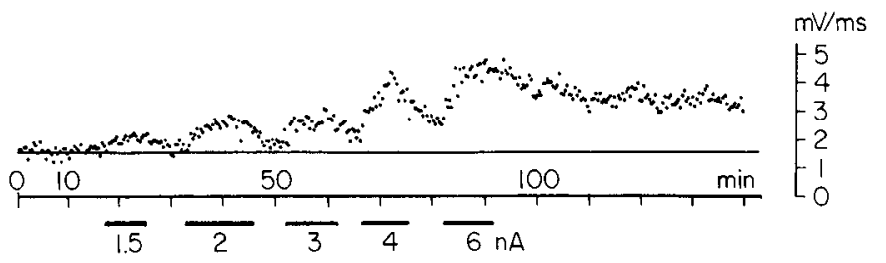

Figure 4. Short- and long-term conjunction effects. Measurements of the initial slope of the intracellular EPSP are shown for a series of responses. During the time indicated by bars, each EPSP was followed by a $100 \mathrm{msec}$ current pulse of indicated magnitude. Note the more transient character of the potentiation obtained at lower current strength. Experiment performed with $0.1 \mathrm{mM}$ PTX in the bath.

average, the EPSP increase $10 \mathrm{~min}$ after being only $34 \%$ of the peak value.

The postconjunction values given above were those obtained following the largest observed increase for each input, not necessarily the largest possible, since often only a single current strength was used (see above). The postconjunction decay of the EPSP increase was, however, not fixed for a given input but depended on parameters such as current strength, number of pairing events, or both. For the input illustrated in Figure 4, the EPSP was subjected to 5 pairing episodes with successively increasing current strength with an interepisode interval of about $5 \mathrm{~min}$. It can be noted that the EPSP increase in response to the lower current strengths (1.5-2 $\mathrm{nA})$ decayed to almost zero within $5 \mathrm{~min}$. Even the responses to 3 and $4 \mathrm{nA}$ showed a considerable initial decay, whereas that to $6 \mathrm{nA}$ decayed more slowly and, thereafter, remained at a high level. The same result was found in 3 additional cells tested in this manner.

\section{Time dependence of the conditioning effect}

As mentioned above, a second input that was alternated with the tested one was unaffected by the current pulse, demonstrating that the induced EPSP increase was not related to general changes in postsynaptic cellular properties and that long intervals $(5 \mathrm{sec})$ between conditioning current pulse and control EPSP did not lead to potentiation. In 16 cells, the current pulse was initially not directly paired with the EPSP but was delayed some $400 \mathrm{msec}$; in none of these cases was any observable increase in EPSP observed. To examine the timing requirement between the EPSP and current pulse in more detail, the current pulse in 11 cells was positioned at one or more intervals ranging from $200 \mathrm{msec}$ after the EPSP to $100 \mathrm{msec}$ before. The EPSP increase reached during the pairing for each interval was then expressed relative to that attained after a final short interval; this is plotted in Figure 5 against the interval between EPSP and current onset. The figure shows that the $100 \mathrm{msec}$ current pulse was ineffective in increasing EPSPs when positioned with its onset $100 \mathrm{msec}$ before the EPSP, i.e., offset of the $100 \mathrm{msec}$ pulse just before EPSP onset ( $n=3$, to the right in the graph; see also input 2, Fig. 1C). On the other hand, when positioned after the EPSP, the pulse was often quite effective at $50-100 \mathrm{msec}$ intervals, increasing EPSP in 8 of 9 cases tested. These increases were 10$50 \%$ of the effect observed at very short intervals. With the longer delays (150-200 msec), no effect was observed in 4 of 6 cases tested and only small increases in the 2 others. This forward-conditioning ability of the current pulse seems somewhat greater than that previously obtained using a brief tetanus as the conditioning stimulus (Gustafsson and Wigström, 1986). However, in that case, only 5 pairing events were performed

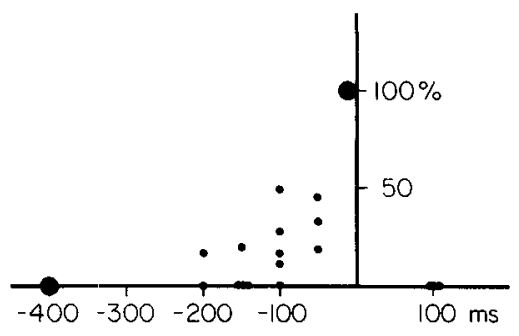

Figure 5. Time dependence of the conditioning effect of a current pulse. The origin of the time axis represents coincidence between the onset of EPSP and current pulse. Negative values $(l e f t)$ indicate the time with which the EPSP onset preceded current onset. The large filled circle at -400 msec signifies that no observable changes in EPSP occurred when this interval was used $(n=16)$. The values at the other intervals (smaller filled circles) represent the maximal EPSP increase found during the conjunction procedure, expressed relative to that found using a short interval (large filled circle at $100 \%$ ).

compared to the 50-60 here, and the EPSP change was not measured during the conjunction but 5 min later.

\section{Occlusion between EPSP increases induced by conjunction and by tetanization}

When a test input had been tetanized prior to pairing with depolarizing current pulses, conjunction effects were much reduced or absent. In the case illustrated in Figure 6 (performed in a PTX-treated slice), input 1 was first tetanized with 10 successive 10-impulse trains, a stimulation that should largely saturate LTP production in PTX-treated slices (Wigström and Gustafsson, 1985b). About $10 \mathrm{~min}$ after the tetanization, the pretetanus EPSP amplitude (intra- as well as extracellular) was reestablished by lowering the stimulation strength ( 30 to $17 \mu \mathrm{A}$; arrow), and the EPSP was paired with a $4 \mathrm{nA}, 100 \mathrm{msec}$ current pulse 45 times. This conjunction episode led to no apparent change in the test EPSP, while a subsequent similar pairing between the current pulse and the other EPSP (input 2) led to a $60 \%$ increase during the conjunction procedure. This result, i.e., a conjunction effect only on the previously untetanized input, was observed in 6 of the 10 cells examined in this manner. In the remaining 4, EPSP increases were observed, but they were small and decayed completely within a few minutes after the conjunction.

We also examined the extent to which the potentiation induced by pairing blocked that evoked by tetanic activation. In the example shown in Figure 6, test input 2 was subjected to additional pairings with pulses also of higher strength (up to 6 $\mathrm{nA}$ ) in order to maximize the conjunction effect. About $10 \mathrm{~min}$ after the conjunction, the input was tetanized with 10 successive 10-impulse trains, leading to a mainly transient EPSP increase. Thus, while the field EPSP was increased by $70 \%$ at $10 \mathrm{~min}$ after tetanization, the corresponding change of the intracellular EPSP was only about $10 \%$. This discrepancy contrasts with the proportional change of field EPSP and intracellular EPSP for input 1 following tetanization, where both returned to baseline upon the same reduction of stimulus strength. A practically identical result to that in Figure 6 was obtained in the other neuron examined in this manner. In 2 other cells, tetanization applied directly after conjunction produced no additional EPSP increase ( $10 \mathrm{~min}$ posttetanus) to that obtained during the conjunction. In 4 additional cells, tetanization was evoked during a prolonged conjunction period (20-30 min), leading to no or small $(<10 \%)$ tetanus-induced intracellular EPSP potentiation 

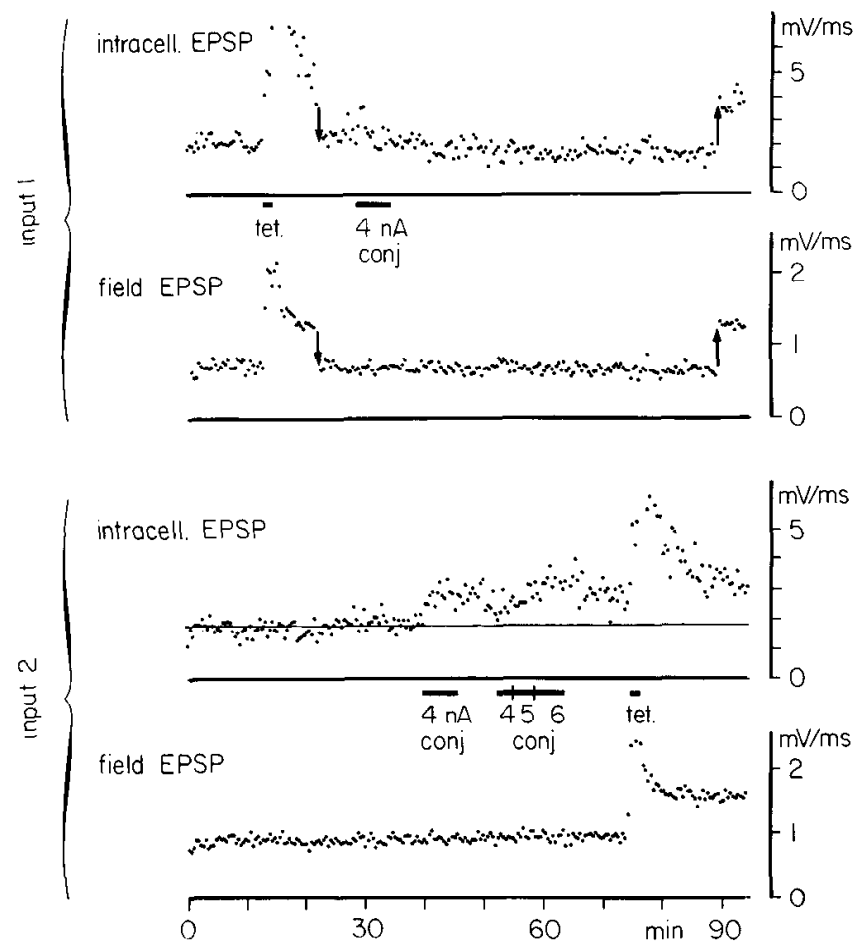

Figure 6. Occlusion between potentiation evoked by homosynaptic tetanization and by conjunction. Measurements of initial slopes of intracellular EPSPs and field EPSPs resulting from activation of input 1 (top) and input 2 (bottom) are shown for a series of responses. Input 1 was first homosynaptically tetanized (tet.) with 10 consecutive 10 -impulse trains (bar). After the tetanization (between the arrows), input 1 was lowered from 30 to $17 \mu \mathrm{A}$. During the time indicated by the bar, each EPSP in response to input 1 was followed by a $4 \mathrm{nA}, 100 \mathrm{msec}$ depolarizing current pulsc. Input 2 was first paired with a depolarizing current pulse (bars) of the indicated magnitude and was subsequently tetanized with 10 consecutive 10-impulse trains. Experiment performed with $0.1 \mathrm{~mm}$ PTX in the bath.

compared to $55-80 \%$ field EPSP increases. As in the case illustrated in Figure 6, tetanization of the input not subjected to prior conjunction produced intracellular EPSP potentiation, and the conjunction itself gave EPSP increases of $80-90 \%$ before the tetanization.

\section{Effect of 2-amino-5-phosphonovalerate (APV) on pairing-induced potentiation}

The potentiation induced by homosynaptic tetanization, as well as by conjunction between single volleys and brief tetani to a separate input, is blocked by the $N$-methyl-D-aspartate (NMDA)receptor antagonist APV (Collingridge et al., 1983; Wigström and Gustafsson, 1984; Gustafsson and Wigström, 1986). Figure 7 shows that the same is true for the potentiation generated by pairing single volleys and depolarizing current pulses. The input tested before APV application (input 1) showed a large and prolonged conjunction-induced EPSP increase, while that tested soon after APV application (input 2) was affected much less. After a $50 \mathrm{~min}$ washout period, a repeated test on input 2 demonstrated the capacity for conjunction-induced potentiation for this input. The same experiment performed in 3 additional cells gave essentially the same result. In these cases, larger drops of APV were applied, which resulted in a complete block of conjunction effects, even transient ones, despite 80-130\% EPSP increases during the conjunction for the inputs tested before

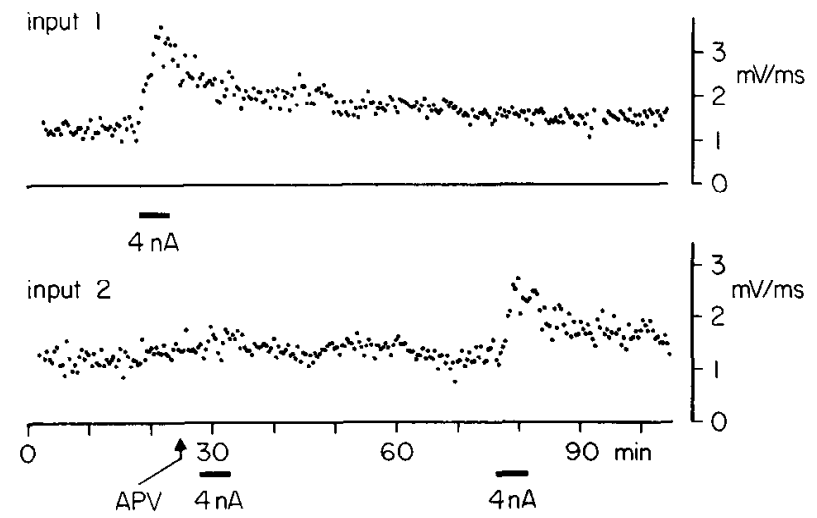

Figure 7. Effect of APV on conjunction-induced EPSP potentiation. Measurements of the initial slope of intracellular EPSPs resulting from activation of inputs 1 and 2 are shown for a series of responses. During the times indicated by bars, each EPSP for the indicated input was followed by a $100 \mathrm{msec}, 4 \mathrm{nA}$ depolarizing current pulse. D-APV (Cambridge Research Biochemicals) was applied as a droplet ( $2 \mathrm{~mm}$ dissolved in the perfusion solution) onto the surface of the slice at the time indicated by an arrow.

application. There was, on the other hand, no recovery from these APV effects when tested about an hour after the application.

\section{Discussion}

Based on experiments using a brief tetanus of separate afferents as conditioning stimulation to induce LTP in single test volley EPSPs, it was suggested that the cooperativity of LTP is due to the dependence of the LTP induction mechanism on postsynaptic depolarization (Gustafsson and Wigström, 1986). The present results strongly support this suggestion by showing that the brief tetanus can be replaced by an intracellularly applied depolarizing current pulse as conditioning stimulus. Thus, pairing the current pulse and a single-volley EPSP results in a longlasting EPSP increase that is specific to the paired EPSP (not affecting an unpaired synaptic input projecting to the same dendritic region), reaches a pcak within 20-30 pairing events, and is blocked by homosynaptic tetanization and by APV. These are the same characteristics that were exhibited by the potentiation obtained using brief tetani as conditioning stimulation, and they suggest, furthermore, that the EPSP increase we observed was the same as that induced by homosynaptic tetanization. Moreover, the finding that homosynaptic tetanization produced little LTP after this pairing procedure suggests that LTP, at least over the time span examined, is controlled by postsynaptic depolarization and does not depend on high-frequency presynaptic activity for induction.

It has been proposed, for a variety of reasons, that LTP is induced as a consequence of opening voltage-sensitive synaptic NMDA-receptor channels that are situated in the spine membrane in close association with the channels that give rise to the potentiated EPSP (Wigström and Gustafsson, 1985a). The present results support this proposal by showing the importance of postsynaptic depolarization for LTP induction. Moreover, the timing requirements for the current pulse with respect to the EPSP, as observed in the present experiments, roughly agree with the proposal. Thus, with the current pulse positioned just before the EPSP, no potentiation was observed; this is as expected, since there would be no interaction between membrane 
depolarization and the synaptic NMDA response in this case. On the other hand, the current pulse could be positioned 50$100 \mathrm{msec}$ (and in some cases even 150-200 msec) after the EPSP and still give rise to some conjunction effect. This forwardconditioning ability, also observed previously when using a brief tetanus as conditioning stimulation, is likely related to the long duration of the NMDA component of the synaptic potential (Gustafsson and Wigström, 1986). When examined at positive membrane potentials, the APV-blocked component of the synaptic potential following a single volley can have a duration of several hundred milliseconds (Wigström and Gustafsson, 1986). Thus, strong membrane depolarization starting even 100-200 msec after the EPSP may interact with synaptically controlled NMDA-receptor channels.

The current pulses that were effective in inducing LTP gave rise to a regular repetitive discharge of somatic action potentials at an average frequency during the pulse exceeding some $60 \mathrm{~Hz}$. However, since the potentiation was also produced after blockade of spike activity by intracellular QX-314, somatic action potential discharge per se was clearly not the critical factor for the conjunction effect (Douglas et al., 1982; Lee, 1983; Gustafsson and Wigström, 1986; however, see Scharfman and Sarvey, 1985). Since the pulses were ineffective when positioned immediately before the EPSPs, it is also unlikely that the conditioning effect was secondary to any changes in ionic concentrations resulting from the action potentials or from the responses appearing after blockade of firing. The simplest interpretation, then, is that the critical conditioning factor is the membrane depolarization produced by the current pulse, as discussed above. Higher discharge rates are associated with a larger average depolarization during the pulse due to the increased number of spikes and to the higher average potential level during the interspike intervals. It may then be assumed, according to the above proposal, that it is this average depolarization that must exceed a certain value to sufficiently affect the ionic flux through synaptic NMDA channels. Current-voltage relations for the response to iontophoretically applied NMDA are also fairly steep in this membrane potential region (Dingledine, 1983), lending a certain credence to this idea.

To account for the input specificity of LTP, it has been suggested that the interaction between synaptic activation and postsynaptic depolarization occurs in the spine membrane (Wigström and Gustafsson, 1985a). This notion is also supported by the finding of a voltage-sensitive, APV-blocked component of the synaptic response to single-volley stimulation that goes in parallel with the non-NMDA component (Wigström and Gustafsson, 1986). The depolarization in the spine may be supplied from several sources: the non-NMDA (and NMDA) channels in the spine itself, synaptic activation of other spines, and the activation of intrinsic voltage-dependent channels in the somatodendritic membrane resulting secondarily from the synaptic activity. In the present study, the homosynaptically induced depolarization (mediated through non-NMDA receptor channels) was not involved since the current pulses were applied after the peak of the somatically recorded EPSPs, i.e., at a time when the depolarization of the spine membrane itself has largely decayed. This suggests that any direct interaction between the homosynaptically induced depolarization and that evoked by the conditioning pulse is not necessary to induce LTP. The heterosynaptic activity was simulated here by a somatically applied current pulse, representing an average action of distributed synaptic inputs from all parts of the neuron. Under these con- ditions, it emerged that LTP was not produced until spike activity was evoked, i.e., until active currents were also involved. The threshold with respect to spike activity was quite high, i.e., the injected current had to generate an average spike frequency exceeding some $60 \mathrm{~Hz}$. It might be expected that this threshold frequency would be lower if the conditioning effect were mediated via excitatory synaptic activation of inputs spatially close to the testcd synapses than by current injection into the soma. However, there is presently no information available on this point since in the extracellular studies, in which a brief tetanus was used as conditioning stimulation, spike activity was not monitored. With respect to a possible effect of inhibitory inputs on the threshold spike frequency, it can be noted that when adjusting for the number of action potentials during the current pulse, there was no obvious difference in potentiation between experiments performed in normal solution and after blockade of inhibition by PTX. Thus, the IPSP (with at least a partly dendritic location) that follows the single-volley EPSP did not have a decisive action on the threshold spike frequency for the conditioning effect. However, it cannot be excluded that larger inhibitory activation could have such action.

The present results should not be taken to suggest that intense spike activity is always necessary for LTP, since they deal only with the situation in which the tested input is activatcd by single volleys. When the tested input is tetanized, there will also be a homosynaptically produced depolarization of the spine membrane, which may be more unrelated to somatic spike activity. In experiments performed by Wigström et al. (1982), somatically applied hyperpolarizing current pulses, while significantly reducing somatic spike activity, did not affect the LTP induced by homosynaptic tetanic activation. This hyperpolarizing current injected during massive excitatory and inhibitory synaptic activation was not likely to have significantly affected the spine membrane depolarization nor, therefore, the LTP induction (for other examples of this type of dissociation between LTP and spike activity, see Bliss and Lomo, 1973; McNaughton et al., 1978; Douglas et al., 1982).

As with the potentiation induced by pairing with a brief tetanus, the potentiation studied here contained a component that decayed within a few minutes. This short-lasting component generally appeared as an initial decay phase following conjunction stimulation, but it was also induced in isolation, e.g., when using low current strength. To what extent this short-lasting component results from the same underlying mechanism as the more prolonged one cannot be determined from the present results. However, the results do demonstrate that there exists a short-lasting potentiation phenomcnon that is controlled by presynaptic activity and postsynaptic depolarization in combination, and that this component has a lower induction threshold than the more long-lasting effect. A linkage between a shortand long-term potentiation agrees with results obtained by $\mathrm{Ra}$ cine et al. (1983). However, the present results show that both potentiation processes observed here are controlled by postsynaptic activity and are therefore not presynaptic postactivation phenomena, as was suggested by those investigators.

Finally, the present results imply that not much additional LTP may be induced by homosynaptic tetanization if the tested EPSP has previously been evoked under conditions in which it was immediately followed by large postsynaptic depolarizations. The absence of tetanus-induced LTP in experiments using cesium-filled intracellular microelectrodes (Haas and Rose, 1984) may then possibly be accounted for on this basis. 


\section{References}

Bliss, T. V. P., and T. Lomo (1973) Long-lasting potentiation of synaptic transmission in the dentate area of the anaesthetized rabbit following stimulation of the perforant path. J. Physiol. (Lond.) 232: 331-356.

Collingridge, G. L., S. J. Kehl, and H. McLennan (1983) Excitatory amino acids in synaptic transmission in the Schaffer collateral-commissural pathway of the rat hippocampus. J. Physiol. (Lond.) 334: 33-46.

Connors, B. W., and D. A. Prince (1982) Effects of local anaesthetic QX-314 on the membrane properties of hippocampal pyramidal neurones. J. Pharmacol. Exp. Ther. 200: 476-481.

Dingledine, R. (1983) N-methyl aspartate activates voltage-dependent calcium conductance in rat hippocampal pyramidal cells. J. Physiol. (Lond.) 343: 385-405.

Douglas, R. M., G. V. Goddard, and M. Riives (1982) Inhibitory modulation of long-term potentiation: Evidence for a postsynaptic locus of control. Brain Res. 240: 259-272.

Eccles, J. C. (1983) Calcium in long-term potentiation as a model for memory. Neuroscience 10: 1071-1081.

Gustafsson, B., and H. Wigström (1986) Hippocampal long-lasting potentiation produced by pairing single volleys and brief conditioning tetani evoked in separate afferents. J. Neurosci. 6: 1575-1582.

Gustafsson, B., M. Galvan, P. Grafe, and H. Wigström (1982) A transient outward current in a mammalian central neurone blocked by 4-aminopyridine. Nature 299: 252-254.

Haas, H. L., and G. Rose (1984) The role of inhibitory mechanisms in hippocampal long-term potentiation. Neurosci. Lett. 47: 301-306.

Hopkins, W. F., and D. Johnston (1984) Frequency-dependent noradrenergic modulation of long-term potentiation in the hippocampus. Science 226: 350-352.

Lee, K. S. (1983) Cooperativity among afferents for the induction of long-term potentiation in the CAl region of the hippocampus. $J$. Neurosci. 3: 1369-1372.
Levy, W. B., and O. Steward (1979) Synapses as associative memory elements in the hippocampal formation. Brain Res. 175: 233-245.

Levy, W. B., and O. Steward (1983) Temporal contiguity requirements for long-term associative potentiation/depression in the hippocampus. Neuroscience 8: 791-797.

McNaughton, B. L., R. M. Douglas, and G. V. Goddard (1978) Synaptic enhancement in fascia dentata: Cooperativity among coactive afferents. Brain Res. 157: 277-293.

Racine, R. J., N. W. Milgram, and S. Hafner (1983) Long-term potentiation phenomena in the rat limbic forebrain. Brain Res. 260: 217-231.

Scharfman, H. E., and J. M. Sarvey (1985) Postsynaptic firing during repetitive stimulation is required for long-term potentiation in hippocampus. Brain Res. 331: 267-274.

Skrede, K. K., and R. H. Westgaard (1971) The transverse hippocampal slicc: A well-defined cortical structurc maintained in vitro. Brain Res. 35: 589-593.

Teyler, T. J., and P. Discenna (1984) Long-term potentiation as a candidate mnemonic device. Brain Res. Rev. 7: 15-28.

Wigström, H., and B. Gustafsson (1984) A possible correlate of the postsynaptic condition for long-lasting potentiation in the guinea pig hippocampus in vitro. Neurosci. Lett. 44: 327-332.

Wigström, H., and B. Gustafsson (1985a) On long-lasting potentiation in the hippocampus: A proposed mechanism for its dependence on coincident pre- and postsynaptic activity. Acta Physiol. Scand. 123: 519-522.

Wigström, H., and B. Gustafsson (1985b) Facilitation of hippocampal long-lasting potentiation by GABA antagonists. Acta Physiol. Scand. 125: $159-172$.

Wigström, H., and B. Gustafsson (1986) Postsynaptic control of hippocampal long-term potentiation. J. Physiol. (Paris) (in press).

Wigström, H., B. L. McNaughton, and C. A. Barnes (1982) Longterm synaptic enhancement in hippocampus is not regulated by postsynaptic membrane potential. Brain Res. 233: 195-199.

Wigström, H., B. Gustafsson, Y.-Y. Huang, and W. C. Abraham (1986) Hippocampal long-term potentiation is induced by pairing single afferent volleys with intracellularly injected depolarizing current pulses. Acta Physiol. Scand. 126: 317-319. 